\title{
NOTIONS OF SYMMETRY IN HUMAN MOVEMENT FOR RECOGNITION
}

\author{
J. B. Hayfron-Acquah ${ }^{1}$, M. S. Nixon ${ }^{2}$ and J. N. Carter ${ }^{2}$ \\ ${ }^{1}$ Kwame Nkrumah University of Science and Technology, Kumasi, Ghana. \\ ${ }^{2}$ University of Southampton, Southampton S017 1BJ, United Kingdom
}

\begin{abstract}
Notions of symmetry are powerful for understanding as they explore relationships in nature for analysis. We describe how symmetry analysis can be used to recognize people by their gait. This approach is reinforced by the view from psychology that human gait is a symmetrical pattern of motion and that symmetrical properties of human movement can indeed be used for human gait analysis. Here, we use gait as a vehicle to investigate both the symmetry of moving objects as provided in our new spatial and spatio-temporal symmetry analyses. We apply these symmetry extractions to a number of databases to demonstrate their potency. A performance analysis shows that using symmetry for gait recognition enjoys practical advantages such as relative immunity to noise, ability to handle missing information and the capability to handle occlusion. The results show that the symmetrical properties of human gait appear to be unique and can indeed be used for analysis and for recognition with recognition rates exceeding 90\%. Best performance is achieved by a spatio-temporal operator, reflecting the view that recognition by gait is not just from body shape, but also by the way the body moves.
\end{abstract}

Keywords: Biometric, Gait, Recognition, Symmetry

\section{INTRODUCTION}

We show how symmetry analysis can expose symmetry of human gait for biometric purposes. As a biometric, gait concerns recognising people by the way they walk. Gait is known to have a number of advantages over other biometrics and notably among these are that its application does not require contact with subjects, it is currently the only biometric applied at a distance and it is difficult to disguise one's gait when walking naturally. One motivation for the increasing demand in gait recognition could be an application scenario involving armed robbers. They usually wear helmets, mask, spectacles and/or gloves thus making it virtually impossible to use any of the other known biometrics such as fingerprint and face. However, aiming not to attract attention, the robbers unconsciously walk or run naturally thus exposing their natural gait. Though it could be argued that physical condition factors such as

116 Journal of Science and Technology, Vol. 29, No. 1, April, 2009 
drunkenness, pregnancy and injuries involving joints can affect an individual's motion, these factors are similar in principle to those affecting other biometrics and should follow after more basic studies on the individuality of gait are complete.

Over the past few years, there has been a steady increase in demand for gait research. The aim of gait recognition is to recognise people by using computer vision analysis on side-view sequences of movement of a person that reveal shape and motion. There have been allied studies of gait that lend support to the view that gait has clear potential as a biometric, notable among these are medical studies, psychological studies, modelling of human motion and tracking people. From these studies, psychologists have suggested that gait is a symmetrical pattern of motion (Cutting et al., 1978) and that humans perceive gait as unique.

\section{MATERIALS AND METHODS}

\section{Early Approaches to Gait Recognition}

Though gait recognition appears to be a fairly new area of research, there are already a number of different approaches. The earliest approach used spatio-temporal analysis where the gait signature was derived from the spatio-temporal patterns of a walking person (Niyogi et al., 1994). The different patterns of the motions of the head and the legs were extracted and processed to determine the motion of the individual's bounding contours from which a five-stick model was fitted. The gait signature was then derived by normalising the fitted model in terms of velocity, by using linear interpolation, recognition rate of $85 \%$ was achieved.

Little et al., (1998) used optical flow to derive the gait signature by analysing the motion content (shape of motion) of a human walking. Generic object-motion characterisation is also another approach where the gait signature is derived from a parametric eigenspace (Murase et al., 1996) and the approach was applied to a database of seven subjects with ten image sequences of each. The recognition rates were $88 \%$ and $100 \%$ for 8 and
16 eigenvectors, respectively. The approach was extended by Huang et al., (1999) who used canonical analysis, a model free approach to reduce the dimensionality of the input data whilst optimising class separability. Recently, Shutler et al., (2000) extended statistical gait recognition via temporal moments. The derived statistics has an intimate relationship to gait, with symmetry properties. Cunado et al., (2003) derived gait signatures from the frequency components of the variations in the inclination of the human thigh. The periodic motion of the thigh during walking was modelled as a pendulum and this again suggests that symmetry analysis is suited to gait recognition. Recent papers by BenAbdelkader et al., (2002), Johnson et al., (2001), Lee et al., (2002) and Tanawongsuwan et al., (2003) support the notion of gait as a potential biometric, especially the notion of self-similarity for recognition.

Most of the current approaches have used small datasets usually up to 30 subjects. We show how symmetry can be used to recognise people by their gait, again using these smaller datasets and using a much larger datasets. We also investigate the performance of symmetry with respect to performance factors including noise, occlusion and missing frames.

\section{Symmetry}

Symmetry in nature is one of the basic features of shapes and objects and is considered a feature that enhances the recognition and the reconstruction of shapes and images (Reisfeld et al., 1995). Every object often gives rise to the human perception of symmetry thereby suggesting symmetry as a fundamental principle. The discrete symmetry operator (Reisfeld et al., 1995) can estimate symmetry without the knowledge of the object's shape. Unlike other feature extraction operators that find a shape by relying on the border of the shape, the symmetry operator locates shapes according to their symmetrical properties. It essentially performs local operations on edge detected images to determine whether a given shape is symmetric or not. 
Though there is good support for gait symmetry, there is actually far more support for gait asymmetry. In a review of support for gait symmetry, Sadeghi et al., (2001), observed that it has been reported in only a few studies using quantitative biomechanics, and that there are not enough studies where a substantial number of subjects participated. On the support for gait asymmetry, Sadeghi et al., concluded that able-bodied gait seems to be naturally asymmetrical and this asymmetrical behaviour could be associated with the different contributions of the lower limb in carrying out propulsion and control task. Therefore, accepting that gait is asymmetrical in the able-bodied population is important for medical studies and the study of physical activities, gait analysis and evaluation. Asymmetry is therefore more potent for gait recognition. Pure symmetry (for example that of simple harmonic motion) is insufficient for recognition by gait as it implies a lack of forward movement.

Recently the notion of symmetry has been applied in a number of application areas, including face recognition and vehicle tracking. Our earlier work (Hayfron-Acquah et al., 2002) has shown that symmetry averaged over a sequence of frames can be used to recognise moving objects together with performance aspects, but only on a small collection of subjects. We now show how we define new measures of symmetry, especially temporal symmetry, to highlight different symmetry contributions. These different approaches are explored by experimental analysis on a selection of gait databases, especially databases which are much larger than earlier works, to explore not only which approach can be best used for analysis, but also explores the effects of covariate factors as well as performance factors, prior to overall conclusions from this work.

\section{Symmetry Extraction}

We extract the symmetry of a walking human subject from a sequence of silhouettes derived from video images. The symmetry operation processes either edge maps (to reduce computa- tional demand, with invariance to global illumination shift). Essentially, images are produced which reflect the aggregated symmetry relationship between pairs of points.

\section{Spatial symmetry}

Our analysis is based on the Discrete Symmetry Operator (Reisfeld et al., 1995). In this, the symmetry relation or contribution, $C(i, j)$ between two (edge) points $P_{i}$ and $P_{j}$ is given by

$C(i, j)=D(i, j) P h_{i, j} I_{i} I_{j} \quad i \neq j$

where $D(i, j)$ and $P h(i, j)$ are the symmetry distance and the phase between the two points respectively. $I_{i}$ and $I_{j}$ are the intensities at the two points. The symmetry distance weighting function, $D(i, j)$, reflects the distance between two different points $P_{i}$ and $P_{j}$, and is calculated as:

$D(i, j)=1 / \sqrt{2 \pi \sigma} \exp \left(-\left(\frac{\left\|P_{i}-P_{j}\right\|-\mu}{2 \sigma}\right)\right), \forall i \neq j$

where $\sigma$ controls the scope of the function. Each value of $\sigma$ implies a different scale thus making it suited to multi-resolution schemes. A large value of $\sigma$ implies large-scale symmetry that gives distant points similar weighting to close points; conversely, a small value of $\sigma$ implies local operation and local symmetry. The focus, $\mu$, was recently introduced by Parsons et al., (1999) to control the focusing capability of the function, hence further improving the scaling possibilities of the symmetry distance function. The addition of focus into the distance weighting function moves the attention of the symmetry operator from points close together to a selected distance.

The logarithm intensity function, $I_{i}$, of the edge magnitude $M$ at point $P_{i}(x, y)$ is given by

$I_{i}=\log \left(1+M_{i}\right)$

where

$M_{i}=\sqrt{M_{x}^{2}(x, y)+M_{y}^{2}(x, y)}$

118 Journal of Science and Technology, Vol. 29, No. 1, April, 2009 
Using the logarithm of magnitude reduces the differences between high gradients or symmetries resulting from weak edges, making the correlation measure less sensitive to very strong edges. In this work, the $M_{x}(x, y)$ and $M_{y}(x, y)$ are the result of convolving the two Sobel templates. The phase weighting function between two points.

$P_{i}$ and $P_{j}$ with edge directions $\theta_{i}$ and $\theta_{j}$, respectively, is:

$P h(i, j)=\left(1-\cos \left(\theta_{1}-\theta_{j}-2 \alpha_{i, j}\right)\right)\left(1-\cos \theta_{1}-\theta_{j}\right) \forall i \neq j$,

where $\alpha(i, j)=\tan ^{-1}\left(\frac{y_{i}-y_{j}}{x_{i}-x_{j}}\right)$

is the angle between the line joining the two points and the horizontal axis. The first factor, (1$\left.\cos \left(\theta_{i}+\theta_{j}-2 \alpha_{i, j}\right)\right)$ makes it possible for maximum symmetry to be achieved when $\left(\theta_{i}-\alpha_{i, j}\right)+\left(\theta_{j}-\alpha_{i, j}\right)=$ $\pi$. This is when the two gradients at the points $P_{i}$ and $P_{j}$ are oriented in the same direction towards each other. This situation corresponds to a dark object on a light background. The second factor, $\left(1-\cos \left(\theta_{i^{-}}-\theta_{j}\right)\right)$ is introduced because the case $\left(\theta_{i^{-}}\right.$ $\left.\alpha_{i, j}\right)=\left(\theta_{j}-\alpha_{i, j}\right)=\pi / 2$ is included in $\left(\theta_{i^{-}} \alpha_{i, j}\right)+\left(\theta_{j^{-}}\right.$ $\left.\alpha_{i, j}\right)=\pi$ when the first factor attains its maximum. This is when the edge directions are normal to the line joining the points $P_{i}$ and $P_{j}$. This situation also corresponds to a light object on a dark background. The combination of factors makes it possible to achieve the same measure for different object reflectance and lighting conditions.

The symmetry contribution value obtained is then plotted at the midpoint of the two points $P_{i}$ and $P_{j}$, that is

$M_{\sigma, \mu}\left(P_{k}\right)=\sum_{(i, j) \in \Gamma\left(P_{k}\right)} C(i, j)$

where $M_{\sigma, \mu}$ is the edge magnitude (obtained using the values of $\sigma$ and $\mu$ ). The $\Gamma\left(P_{k}\right)$ defines the set of values for the coordinates $(i, j)$ and the direction of the contribution from $P_{i}$ and $P_{j}$ is given by

$\varphi(i, j)=\frac{\left(\theta_{i}+\theta_{j}\right)}{2}$
Fig. 1 shows two different image points $P_{i}$ and $P_{j}$, the symmetry contribution of the two points and the direction of symmetry. The symmetry transform as discussed here detects reflectional symmetry. It is invariant under 2-Dimensional (small angle) rotation and translation transformations and under change in scale (Reisfeld et al., 1995), and as such has potential advantage in automatic gait recognition.

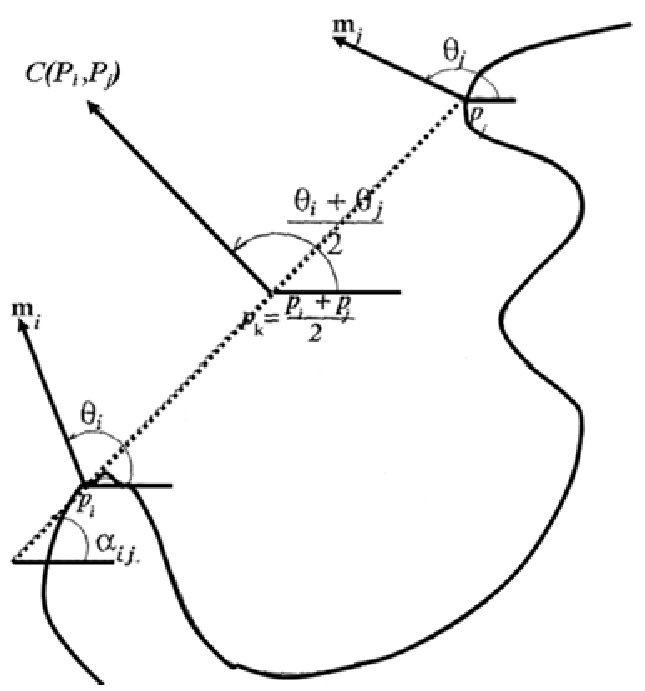

Figure 1: Symmetry Contribution Calculation

\section{Spatio-temporal and extended spatio-temporal symmetry}

The spatio-temporal and the extended spatiotemporal symmetry extractions are attempts to include temporal information in the symmetry computation, thus we extract symmetry between pairs of (edge) points that are separated spatially (within an image) and temporally (between two images). The symmetry contribution between pairs of image points is given by

$C(i, j)=\left(D_{s}(i, j)+w D_{t}(i, j)\right) P h_{i, j} I_{i} I_{j}$

where $D(i, j), P h(i, j), I_{i}$ and $I_{j}$ have the same meaning as before. $D_{s}$ and $D_{t}$ are the spatial and the temporal symmetry distance respectively. $w$ is a space/time weighting function that makes it 
possible to control the contributions of spatial and temporal information in a symmetry map. The symmetry distance is given by

$$
\begin{array}{r}
D(i, j)=\frac{1}{\sqrt{2 \pi \sigma_{S}}} \exp \left(-\left(\frac{\left\|P_{i}^{t}-P_{j}^{t}\right\|-\mu_{S}}{2 \sigma_{S}}\right)\right) \\
+\frac{w}{\sqrt{2 \pi \sigma_{T}}} \exp \left(-\left(\frac{\left\|P_{i}^{t}-P_{j}^{t-1}\right\|-\mu_{S}}{2 \sigma_{T}}\right)\right)
\end{array}
$$

where $\sigma_{S}$ and $\sigma_{T}$ controls the scope of the spatial and temporal distance weighting function respectively. $m_{S}$ and $m_{T}$ are their respective focus. The first part of equation (10) specifies the spatial component and the second factor the temporal. Here, we assume variance and focus are the same for time and space, thus $\sigma_{T}=\sigma_{S}=\sigma$ and $m_{S}=m_{T}$ $=m$.

Similarly, for the extended spatio-temporal symmetry approach, each symmetry map is derived from three successive image frames in a sequence as shown in Fig. 2. The spatial symmetry contribution is computed for each of the frames and the temporal symmetry contribution for the

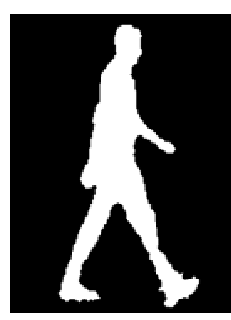

(a) $F_{t-1}$

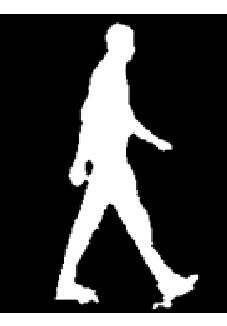

(b) $F_{t}$

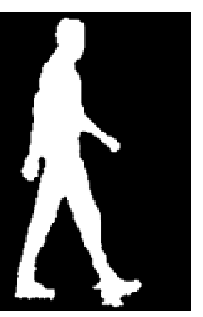

(c) $F_{t+1}$
Figure 2: Three Successive Image Frames of a Gait Sequence

pairs $\left(F_{t-1}\right.$ and $\left.F_{t}\right)$ and $\left(F_{t}\right.$ and $\left.F_{t+1}\right)$. If $D\left[F_{A}(i, j)\right]$ and $D\left[F_{A, B}(i, j)\right]$ are the spatial symmetry distance from image frame $F_{A}$ and the temporal symmetry distance from the image frames $F_{A}$ and $F_{B}$ respectively of the image points $P_{i}$ and $P_{j}$ then the symmetry distance weighting function is obtained by

$$
\begin{array}{r}
D(i, j)=D_{s 1}\left[F_{t-1}(i, j)\right]+D_{s 2}\left[F_{t}(i, j)\right] \\
+D_{s 3}\left[F_{t+1}(i, j)\right]+w\left(D_{t 1}\left[F_{t, t-1}(i, j)\right]\right. \\
\left.+D_{t 2}\left[F_{t, t+1}(i, j)\right]\right)
\end{array}
$$

For simplicity, let $s=s 1=s 2=s 3$ and $t=t 1=t 2$ then the extended spatio-temporal distance weighting function is given by equation (12)

$$
\begin{aligned}
D(i, j) & =\frac{1}{\sqrt{2 \pi \sigma_{t}}}\left(\exp \left(-\frac{\left\|F_{t-1}(i)-F_{t-1}(j)\right\|-\mu_{s}}{2 \sigma_{s}}\right)\right. \\
& +\exp \left(-\frac{\left\|F_{t}(i)-F_{t}(j)\right\|-\mu_{s}}{2 \sigma_{s}}\right) \\
& \left.+\exp \left(-\frac{\left\|F_{t+1}(i)-F_{t+1}(j)\right\|-\mu_{s}}{2 \sigma_{s}}\right)\right) \\
& +\frac{1}{\sqrt{2 \pi \sigma_{t}}}\left(\exp \left(\frac{\left\|F_{t}(i)-F_{t-1}(j)\right\|-\mu_{t}}{2 \sigma_{t}}\right)\right) \\
& +\exp \left(-\frac{\left\|F_{t}(i)-F_{t+1}(j)\right\|-\mu_{t}}{2 \sigma_{t}}\right)
\end{aligned}
$$

Equation (12) therefore replaces Equation (2). Note that $2 \leq \mathrm{t} \leq \mathrm{n}$ where $\mathrm{n}$ is the number of image frames.

\section{Temporal symmetry}

We extract temporal symmetry in a way similar to the spatio-temporal approach discussed above. We used the same distance weighting function with a little modification that is by omitting the $w$ and the spatial component of Equation (8). This gives the temporal symmetry distance weighting function as

$$
\begin{aligned}
& D(i, j)=F D\left\|F_{t}(i)-F_{t-q}(j)\right\| \\
& \left.=\frac{1}{\sqrt{2 \pi \sigma}} \exp -\frac{\left\|F_{t}(i)-F_{t-q}(j)\right\|-\mu}{2 \sigma}\right)
\end{aligned}
$$


where symbols have their usual meanings. $F_{t-q}(i)$ is displaced $q$ frames from $F_{t}(j)$ where $F_{t}(j)$ and $F_{t-q}(j)$ are not necessarily at the same spatial position. For the purpose of this work, we used $q=1$.

\section{Deriving gait signatures}

In this work, symmetry maps are derived from silhouettes. First, the background image is computed. For each image frame in an image sequence, the background is subtracted from it to obtain the image silhouette. The silhouette is first thresholded so as to set all points below a chosen threshold to zero while leaving the others unchanged. The edge map of the silhouette is then derived by applying the Sobel operator. The symmetry operator is then applied to each edge map in a sequence to obtain their corresponding symmetry maps.

Having generated the symmetry maps of all images in a sequence, they are then averaged, that is

$$
G S_{i}=\frac{1}{n} \sum_{j=1}^{n} S_{j}
$$

where $n$ is the number of symmetry maps in a sequence, $S_{j}$ is a symmetry map and $G S_{i}$ is a gait signature of image sequence $i$.

\section{Subject classification}

The $k$-nearest neighbour approach is used to classify the subjects. Here we used $k=1$ and $k=3$. It must be noted that we could have used better classifiers but the k-NN is a good basic technique offering capability for comparison with other classifiers. It is worth mentioning that all those with whom we compared our results used the k$\mathrm{NN}$ for classification. The feature vectors are obtained first by taking the magnitude of the Fourier transform of the gait signatures and then lowpass filtered to reduce sensitivity to highfrequency components. The Fourier transform is used here for reasons of shift invariance and access to frequency components. If $G S$ is a gait signature, then the low-pass filtered feature space, $F S$ is given by
$F S^{\prime}(u, v)=\left\{\begin{array}{l}S F(u, v) \text { if }\left(u^{2}+v^{2} \leq R^{2}\right) \\ 0 \quad \text { otherwise }\end{array}\right.$

Where $u$ and $v$ are the coordinate of a point in the low pass filtered image and $R$ defines the region within which points are selected.

Different cut-off frequencies (covering 0.1-100\% of the Fourier data) were used to determine the appropriate number of Fourier components (Hayfron-Acquah, 2003). For purposes of classification or recognition, the similarity differences, $S D$ between the Fourier descriptions of the gait signatures are then calculated using Euclidean distance, that is, for gait signatures $G S_{i}$ and $G S_{j}$, we compute

$S D_{i, j}=\sum\left\|F S_{x, y}^{i}-F S_{x, y}^{j}\right\|$

where $F S_{x, y}^{i}$ and $F S_{x, y}^{j}$ are the Fourier coefficients of the gait signatures $i$ and $j$ at coordinate $(x, y)$ respectively.

\section{RESULTS AND DISCUSSION}

A number of databases were used in this work. Some of them were used to enable us make comparisons of our results with those that were obtained by others using a different approach, others enabled us to carry out performance analysis related to gait. The main databases were captured at the Carnegie Mellon University, CMU (Gross et al., 2001) and University of Southampton (SOTON) for the Human Identification at a Distance (Human ID) (Shutler et al., 2002) research program.

In the CMU databases the subjects performed four different walk patterns, namely slow walk, fast walk, inclined walk and walking with a ball (Gross et al., 2001). The databases contain the same twenty five (25) subjects filmed walking on an indoor treadmill. Six different cameras at six different positions were used in the filming of the subjects thus giving six different walking views. For the purposes of this work, we selected two of 
the different views that were made available at the time of this work, that is fronto-parallel and oblique (at 45 degrees to the camera view). Thus four different databases from CMU were used here. For the slow walk, the treadmill was adjusted to walking speed of 2.06 miles per hour and for the fast speed it was set to 2.86 miles per hour. We used four image sequences of each subject from each database. Each image sequence contains one gait cycle.

The SOTON'01 consists of 50 subjects, with 4 sequences of each subject giving a total of 200 image sequences. The new SOTON'02 consists of 114 subjects, with 4 image sequences each, with two directions of walk, left to right and right to left (Shutler et al., 2002). The subjects in SOTON'01 form a small part of SOTON'02. For SOTON'02 dataset, we used the silhouette information derived via chromakey analysis as input to our approach. The database containing subjects walking in both directions was obtained by combining the databases of the left to right walk and the right to left walk. Thus, before taking the Fourier transform, the right to left walk gait signatures are all mirrored so that all image sequences in the combined database will have all subjects walking from left to right. Table 1 shows the recognition rates obtained from the databases using the spatial symmetry approach. It is encouraging that the high recognition rates by this and by other techniques have been maintained with a much larger number of subjects. It is worth noting that the by chance classification rate is naturally much lower with the increase in the number of the subjects, whilst the recognition rate remains the same. The other symmetry formulations might show different advantages and performances, as we consider next.

Unless otherwise stated, the values of $\sigma$ and $\mu$ are 27 and 90 respectively. This combination of values ensured that distant points are giving more weighting.

\section{Performance analysis}

For various symmetry analyses to assist in the advancement of gait recognition, they need to
Table 1: Initial Results Obtained from the SOTON'02 database

\begin{tabular}{|c|c|c|c|}
\hline \multirow{2}{*}{$\begin{array}{l}\text { Direction } \\
\text { of walk }\end{array}$} & \multirow{2}{*}{$\begin{array}{c}\text { No. of } \\
\text { Subjects }\end{array}$} & \multicolumn{2}{|c|}{$\operatorname{CCR}(\%)$} \\
\hline & & $K=1$ & $k=3$ \\
\hline Left-Right & 114 & 95.6 & 90.8 \\
\hline Right-Left & 114 & 94.1 & 87.5 \\
\hline Both & 114 & 95.0 & 90.7 \\
\hline
\end{tabular}

perform well under different environments and conditions. In this regard, many published recognition approaches address few factors of practical consequence. It is important to know the conditions under which the gait recognition problem is solvable, the important factors affecting a person's gait and the research approaches that appear promising for improving the performance of gaitbased recognition (Johnson et al., 2001). We also need to know how well our approaches compare with some of the existing approaches, using a common database. With these points in mind, we have evaluated the performance of the symmetry operator by looking at (i) gait factors such as the effect of walking speeds and the direction of walking and (ii) performance factors or generalisation capabilities such as addition or missing spatial data, missing image frames, noise, different image resolutions, direction of walk and different view angles.

It must be noted that the tests on performance and generalisation capability were carried out after the extraction of the silhouette from the sequence. This is because it is difficult to add noise that accurately mimics real-world scenarios so we aimed to look at noise and occlusion in the worse case. Moreover, with powerful noise removal algorithms, noise can easily be reduced to minimum in most cases so noise analysis by inverting points on the extracted silhouette appears to be reasonable.

\section{Gait factors}

Effect of speed on gait by symmetry analysis

We investigated the effect of speed on gait by our methods using the CMU databases. Here, the sub- 
jects were made to perform four different walk patterns namely slow walk, fast walk and inclined walk (Gross et al., 2001). These databases contain the same twenty five subjects filmed walking on an indoor treadmill. We used four image sequences of each subject in each database. Each image sequence contains one gait cycle. From now on, we shall refer to the four databases as $S 37, S 45$ for slow walking filmed fronto-parallel and at 45 degrees to the camera respectively. Also, F37 and F45 refer to the fast walking for filming fronto-parallel and at 45 degrees to the camera respectively.

A gallery and a probe sets are used for this analysis. A gallery is set of gait signatures representing subjects in a database. The unknown gait signatures to be recognised constitute the probe set and there is no reject class. Every signature in the probe set has a corresponding signature in the gallery set and that these represent gait signatures from different image sequences of the same subject.

For example, we used S37 to probe F37 and vice versa as these two databases contain image sequences for which the subjects were filmed fronto -parallel. Similarly, S45 is used to probe F45 and vice versa as both of these databases contain image sequences where the subjects were filmed walking at an oblique angle of 450 to the camera. Table 2 shows the recognition rates obtained by applying all the symmetry extraction techniques described in section 2 .

From the results shown in Table 2 for the different approaches, the spatial and spatiotemporal formulations offer the best performance. The probing of the slow-walk data with the fast-walk data shows that the performance of the symmetry operator is not affected very much by the speed at which subjects walk. This is due to the averaging within the symmetry calculations and by its calculation using frames within one period of a gait cycle. This may be very useful in real application as people do not normally walk at constant speeds in carrying out their daily activities.

\section{Effect of different view angle on symmetry analysis}

To investigate the effect of different view angles, we used the CMU database. Here, for both walking speeds, we used the fronto-parallel (Slow37 and Fast37) databases as the probe and the oblique view (Slow45 and Fast45) databases as the gallery. It must be noted that at the time of this work, we could not obtain databases in which the subjects were filmed at smaller view angles. The only database we could use to carry out this test was the CMU database in which the subjects were filmed fronto-parallel and at a view angle of 45 degrees. By using the fronto-parallel databases to probe oblique databases and vice-versa, we obtained recognition rates of less than $15 \%$ for all the approaches. It is therefore clear that at a view angle of 45 degrees, it is almost impossible to recognise a subject from his/her fronto-parallel video footage by symmetry without viewpoint correction but the change in view is rather severe. Further tests will be required to ascertain the view angle at which a subject can still be recognised from a fronto-parallel video footage.

Table 2: Recognition rates for different symmetry approaches

\begin{tabular}{lcccccccc}
\hline \multirow{2}{*}{$\begin{array}{l}\text { Probe vrs } \\
\text { Gallery }\end{array}$} & \multicolumn{2}{c}{ S37 VRS F37 } & \multicolumn{2}{c}{ S45 VRS F45 } & \multicolumn{2}{c}{ F37 VRS S37 } & \multicolumn{2}{c}{ F45 VRS S45 } \\
& $\mathbf{k = 1}$ & $\mathbf{k = 3}$ & $\mathbf{k}=\mathbf{1}$ & $\mathbf{k}=3$ & $\mathbf{k}=\mathbf{1}$ & $\mathbf{k}=3$ & $\mathbf{k}=\mathbf{1}$ & $\mathbf{k}=3$ \\
\hline Spatial & 75.0 & 76.0 & 71.0 & 70.0 & 70.0 & 68.0 & 76.0 & 73.0 \\
Spatio-Temporal & 75.0 & 74.0 & 70.0 & 69.0 & 71.0 & 67.0 & 75.0 & 72.0 \\
Temporal & 68.0 & 65.0 & 70.0 & 65.0 & 67.0 & 65.0 & 76.0 & 69.0 \\
EST & 70.0 & 68.0 & 69.0 & 69.0 & 73.0 & 67.0 & 77.0 & 68.0 \\
\hline
\end{tabular}


Table 3: Effect of View Angle on Symmetry Analysis using the CMU Databases

\begin{tabular}{lcccccccc}
\hline \multirow{2}{*}{ Method } & \multicolumn{3}{c}{ Fronto-Parallel Database } & \multicolumn{3}{c}{ Oblique Angle Database } \\
& \multicolumn{2}{c}{ Slow walk } & \multicolumn{2}{c}{ Fast walk } & \multicolumn{2}{c}{ Slow walk } & \multicolumn{2}{c}{ Fast walk } \\
& $\mathbf{k = 1}$ & $\mathbf{k = 3}$ & $\mathbf{k = 1}$ & $\mathbf{k = 3}$ & $\mathbf{k = 1}$ & $\mathbf{k = 3}$ & $\mathbf{k = 1}$ & $\mathbf{k = 3}$ \\
\hline Spatial & 100.0 & 100.0 & 100.0 & 99.0 & 100.0 & 100.0 & 100.0 & 99.0 \\
Spatio-Temporal & 100.0 & 100.0 & 100.0 & 99.0 & 100.0 & 100.0 & 100.0 & 99.0 \\
Temporal & 100.0 & 100.0 & 100.0 & 98.0 & 100.0 & 100.0 & 100.0 & 99.0 \\
EST & 100.0 & 100.0 & 100.0 & 99.0 & 100.0 & 100.0 & 100.0 & 98.0 \\
\hline
\end{tabular}

It is clear from Table 3 that if all subjects in a database are filmed walking from the same view angle then the view point has no statistical significant effect on the recognition. Comparing the results of fronto-parallel and the oblique in Table 3 , it can be seen that the recognition rates for the fronto-parallel slow walk and that of the oblique view are about the same. However, there are only small differences in the corresponding rates for the fast walk. The similarities in the overall rates show that for a complete gait cycle, gait appears to be invariant to speed. In future, we will apply the techniques to databases of smaller view angles and make the approaches view invariant.

\section{Performance factors and generalisation capa- bility}

The performance factors aim to indicate likely performance capability with practical video imager. As such, we sought to mimic factors including time lapse imagery, occlusion, noise and low resolution. These factors are often found in application data where time lapse imagery reduces the number of frames stored, subjects can be occluded from the camera's field of view and the video imagery is often of poor quality and/or at low resolution. The last of these factors is one of gait's unique attributes in that it has the potential to achieve recognition even when other biometric data is at too low resolution when other biometric data fail to be useful. Sample images for each of these effects are given in Fig. 4.

Omitting frames

The evaluation, aimed to simulate time lapse, was done by considering a consecutive number of image frames of a sequence as missing, i.e. by omitting them from the computation of symmetry.

We started by omitting every other frame, then by omitting every two successive frames, and continued up to the omission of 8 consecutive frames, each time starting from the first image frame in the sequence. This means we considered about $87 \%$ of the image frames as missing for the omission of 8 consecutive frames. This was applied to both the old SOTON used in (Hayfron-Acquah et $a l ., 2003)$ and the SOTON'02 databases. For the old SOTON database, the missing frames approach was applied to only the test data. Fig. 4(d) shows image frames left after considering every 8 consecutive frames as missing.

The recognition rates obtained from the different techniques using $k=1$ and $k=3$ respectively, on the 114 subject SOTON '02 database show that by having similar image frames missing in all image sequences, recognition rates are not much affected depending on the percentage of frames missing. It was clear that by considering up to $75 \%$ of the image frames as missing (that is using only 3 image frames on average) recognition rates of over $60 \%$ are obtained for both $k=1$ and $k=3$. We also see that by increasing the number of missing frames from $50 \%$ to about $85 \%$ does not lead to drastic fall in recognition rate. The average number of image frames in a sequence is about 28 .

\section{Addition or removal of spatial data}

The evaluations were done on both the old SOTON and the SOTON'02 databases by masking 
tion of spatial data whilst an object with a different colour from the bar illustrate omission of data. By omitting spatial data, we reduce the effect of the main axis of symmetry resulting from the human body with the resulting symmetry map being

(a) addition and removal of spatial data

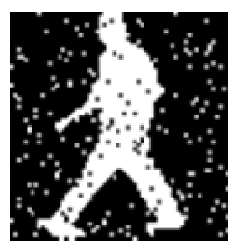

$10 \%$

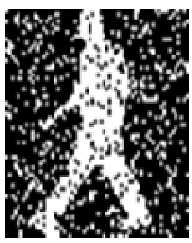

$30 \%$

$50 \%$

$70 \%$

(b) Images showing percentage of artificial noise added to the silhouettes

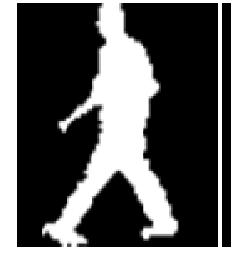

$64 \times 64$

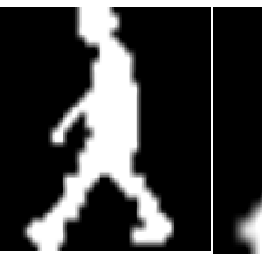

$32 \times 32$

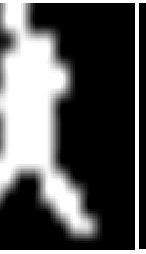

$16 \times 16$

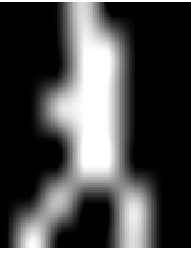

$8 \times 8$ (c) different resolution images (all images have been enlarged to same size)

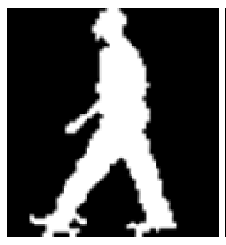

Frame 1

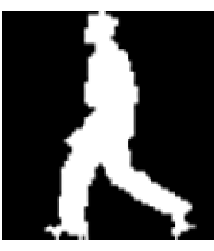

Frame 9

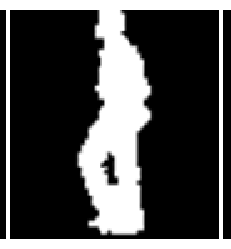

Frame 17

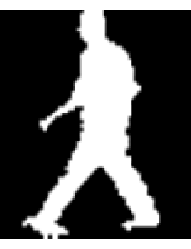

Frame 25 (d) Image frames left after considering sequence of eight consecutive frames as missing

Figure 4: Sample Images used in Performance Analysis

with a rectangular bar of different widths of up to 35 pixels in steps of 5 in each image frame at the same position. These tests were done to see the effect of removing or adding spatial data on the recognition rates. If the object has the same colour as the bar, then that illustrates addiderived mainly from the legs and the arms which are the main parts of the human body associated with gait.

For the old SOTON database, the masking was applied to only the test subject with bar sizes of 5, 10 and 15 . The area masked was on the average, 13.2, 26.3 and $39.5 \%$ of the image silhouette in each image frame. The bar either had the same colour as the image silhouette or as the background. The masking was applied before the Sobel operator. Examples are shown in Fig. 4(a). In both cases, recognition rates of $100 \%$ were obtained for a bar size of 5 pixels for both $k=1$ and $k=$ 3. Masking with a black bar of 10 pixels failed but the masking with white bar of 10 pixels gave correct recognition for $k=$ 3 and not $k=1$. For bar sizes of 15 and above, the test data could not be recognised as the subject is occluded in almost all the image frames in the sequence. Here we used the spatial symmetry approach.

For the SOTON'02 database, the masking was applied to all image sequences in the database. The results obtained were very encouraging as recognition rates of over $70 \%$ were obtained for all techniques, with bar sizes of up to 25 pixels, that covers up to about $80 \%$ of the silhouette in most of the image frames. Here, we used spatial, spatio-temporal, extended spatiotemporal and temporal symmetry approaches. Generally, the fall in recognition rates is gradual with increasing bar width showing how the approaches can tolerate addition and removal of spatial data. 


\section{Noise}

The symmetry operator is able to handle noise by the averaging associated with its evidence gathering and our experimentation has confirmed that using symmetry can tolerate some, but naturally not excessive, additive salt and pepper noise. We added $10 \%$ to $70 \%$ (inclusive) of salt and pepper noise to each image frame of the test subject and compared the resulting signature with those of the training subjects in the database. The noise is added before the Sobel operator. Fig. $4 \mathrm{~b}$ shows samples of the noise levels used. The evaluation was carried out under two different conditions.

For the first condition, we used the same values of $\sigma$ and $\mu$ (see Equation 2) as earlier. With a noise level of $5 \%$, the recognition rates for both $k$ $=1$ and $k=3$ were $100 \%$. With $10 \%$ added noise, the test subject could still be recognised correctly for $k=1$ but not for $k=3$, i.e. CCR of $100 \%$ and $0 \%$ respectively. With added noise levels of $20 \%$ and above, the test subject could not be recognised for both $k=1$ and $k=3$.

For the second condition, the values of $\sigma$ and $\mu$ were made relatively smaller (2 and 10 respectively). The recognition rates were not affected for both $k=1$ and $k=3$ for added noise levels even exceeding $60 \%$ The reduction in the values of $\sigma$ and $\mu$ was to ensure that closer points are given higher distance values than the distant points. This is because the distribution of the noise resulted in having most pairs of the noise as the distant points (Hayfron-Acquah et al., 2003).
The above test showed that if we have noisy subjects to recognise from a database of 'clean' subjects, then we can achieve very good recognition rates. A further test was carried out by adding different noise levels to all image sequences in the SOTON'02 database. Table 5 shows the recognition rates on the different approaches by adding different amounts of artificial noise to the image sequences.

For noise levels up to $30 \%$, the increase in recognition rates is on average about $25 \%$ for both values of $k$ which is very encouraging. This confirms our earlier observation that using Equation 18 rather than equation 8 improves the performance of the symmetry projection. We can deduce from the results that all our approaches can tolerate noise to a large extent. With a very noisy data $25 \%$ can be considered as an acceptance level of recognition.

\section{Low resolution and scaling}

Investigation was also conducted on low resolution images using the SOTON'02 database in which the subjects were walking from right to left. For each image resolution, all image frames in the database were resized to the same size. This was done to simulate both poor images and scaling. We did this by resizing (scaling) the original image sequences before deriving the corresponding gait signature. Fig. 4(c) shows the four different image sizes used, that is $64 \times 64,32$ $\times 32,16 \times 16$ and $8 \times 8$ pixels. The images in the Fig. 4(c) have been enlarged to the same size to

Table 5: effect of noise using SOTON'02 database

\begin{tabular}{|c|c|c|c|c|c|c|c|c|}
\hline \multirow{3}{*}{ Symmetry approach } & \multicolumn{8}{|c|}{ NOISE LEVEL } \\
\hline & \multicolumn{3}{|c|}{$10 \%$} & \multicolumn{2}{|c|}{$20 \%$} & \multirow{2}{*}{$\begin{array}{r}30 \% \\
k=3\end{array}$} & \multicolumn{2}{|c|}{$40 \%$} \\
\hline & $k=1$ & $\mathbf{k}=\mathbf{3}$ & $k=1$ & $\mathbf{k}=\mathbf{3}$ & $k=1$ & & $k=1$ & $k=3$ \\
\hline Spatial & 75.0 & 61.6 & 48.9 & 32.7 & 27.4 & 10.8 & 14.3 & 3.3 \\
\hline Spatio-temporal & 75.9 & 61.8 & 48.0 & 30.3 & 26.8 & 10.5 & 15.1 & 4.4 \\
\hline Temporal & 72.8 & 55.3 & 44.7 & 28.3 & 31.6 & 14.3 & 14.5 & 5.0 \\
\hline EST & 71.3 & 54.0 & 42.5 & 24.3 & 23.5 & 6.6 & 11.8 & 4.0 \\
\hline
\end{tabular}

126 Journal of Science and Technology, Vol. 29, No. 1, April, 2009 
reveal the loss of detail. It can be seen that as the images are made smaller, a considerable amount of information is lost. In (Hayfron-Acquah et al., 2002, 2003), only the test subject was resized. This showed that even in that case, recognition rates of over $95 \%$ could still be achieved for image sizes up to $16 \times 16$ pixels. Table 6 shows the earlier results (Hayfron-Acquah et al., 2002, 2003).

Images are usually normalised to $64 \times 64$ to reduce computational demand. Reduction in resolution by half to $32 \times 32$ changes recognition performance little. Using the same values of $s$ and $m$, recognition rates of over $90 \%$ were obtained for $k=$ 1 and over $80 \%$ for $k=3$ for the $32 \times 32$. In fact, further reduction to $16 \times 16$ is where no other biometric could be perceived, but where recognition by gait still occurs to some extent (one far better than random performance).

For the 16 by 16 images, recognition rates of over $80 \%$ and $70 \%$ were obtained for $k=1$ and $k=3$

Table 6: Recognition rates from low resolution images using spatial approach on old SOTON database

\begin{tabular}{lccc}
\hline Image & \#Subj. & \multicolumn{2}{c}{ Classification Rate } \\
Size & (\#Seq.) & $\mathbf{~ k = 1}$ & $\mathbf{k = 3}$ \\
\hline $64 \times 64$ & $28(4)$ & 97.3 & 96.4 \\
$32 \times 32$ & $28(4)$ & 97.3 & 92.9 \\
$16 \times 16$ & $28(4)$ & 84.0 & 67.0 \\
$8 \times 8$ & $28(4)$ & 57.1 & 33.0 \\
\hline
\end{tabular}

respectively. For the $8 \times 8$ images, the recognition rates were below $50 \%$ for both values of $k$. Looking at the image quality of the silhouette in column 3 of Fig. 4(c), it could be argued that the symmetry operator is able to handle low resolution images or scaling to a large extent. This is due to the averaging associated with the symmetry operator. Table 7 shows the results obtained after applying the different image resolution to all image sequences in the SOTON'02 database.

Our results are contingent on an intimate relationship between gait and the symmetry operator. Essentially, we can only assess broad trends and give a basic comparable performance on gait. We suggest these performance factors should generalise to application of the symmetry operation for this type of analysis.

\section{CONCLUSION}

The aim of this paper is to support the psychology view, and our earlier work, that the symmetry of human motion can indeed be used for recognition. This work has shown that human gait appears to have distinct symmetrical properties that can be extracted for recognition. The symmetry operation essentially forms an accumulator of points, which are measures of the symmetry between image points to give a symmetry map. This has been formulated to highlight different contributions to the overall symmetry, including spatial, spatiotemporal and temporal symmetry. By these operators, and the use of the Discrete Fourier Transform and a basic nearest-neighbour classifier, the results have produced a recognition rate

Table 7: Recognition Rates on Low Resolution Images using SOTON'02 Databases

\begin{tabular}{lcccccc}
\hline \multirow{2}{*}{ Symmetry Approach } & \multicolumn{2}{c}{32 X 32 } & \multicolumn{2}{c}{$\mathbf{1 6}$ X 16 } & \multicolumn{2}{c}{$\mathbf{8 X ~ 8}$} \\
& $\mathbf{k}=\mathbf{1}$ & $\mathbf{k = 3}$ & $\mathbf{k = 1}$ & $\mathbf{k = 3}$ & $\mathbf{k = 1}$ & $\mathbf{k = 3}$ \\
\hline Spatial & 93.4 & 88.2 & 87.9 & 75.2 & 39.7 & 18.2 \\
Spatio-temporal & 94.3 & 88.2 & 87.9 & 75.7 & 38.8 & 19.3 \\
Temporal & 91.7 & 84.9 & 85.1 & 72.8 & 39.3 & 17.8 \\
EST & 93.4 & 88.2 & 88.4 & 76.1 & 38.8 & 20.4 \\
\hline
\end{tabular}


of $97.3 \%$ on a database much larger than any used before. The results showed that the different forms of symmetry discussed can do well in real application scenarios. On these data, the new spatio-temporal operator shows consistently the best performance emphasising that recognition by gait involves not just shape, but also motion. Our new version of projective symmetry has advantageous performance in terms of recognition capability and generalisation than its previous formulation. We believe recognition rates can still be improved by parameter tuning, especially since only basic forms of the new operators have been used here.

In future, we will aim to find alternative and an improved method of incorporating temporal information in our symmetry calculations. We will also consider radial distortion, and this is likely to improve performance more. Though the SOTON'02 database is the largest contemporary database of its kind, we will seek to evaluate our approaches on much larger databases. So far, we have used mainly indoor databases where the environments are usually controlled. In future we aim to use outdoor data. The approaches discussed above appear not to be view invariant and this also will be considered in our further work.

\section{ACKNOWLEDGEMENTS}

We gratefully acknowledge support by the European Research Office of the US Army under Contract No. N68171-01-C-9002.

\section{REFERENCES}

BenAbdelkader, C., Cutting, J., and Davis, L. (2002). Motion-based Recognition of People in EigenGait Space. Proceedings of the 5th IEEE on Automatic Face and Gesture Recognition, 20-21 May 2002, Washington, D.C., USA: $378-384$.

Cunado, D., Nixon, M. S. and Carter, J. N. Carter (2003). "Automatic Extraction and Description of Human Gait Models for Recognition Purposes", Computer Vision and Image Understanding, 90(1): 1-41.
Cutting, J. T., Proffitt, D. R. and Kozlowski, L. T. (1978). "A biomechanical invariant for gait perception”, The Journal of Experimental Psychology: Human Perception and Performance, 4(3): 357-372.

Gross, R. and Shi, J. (2001). The CMU motion of body (MOBO) Database. Technical Report, CMU-RI-TR-01-18 by R. Gross and J. Shi, Robotics Institute, Carnegie Mellon University, Pittsburgh, PA, June, 2001.

Hayfron-Acquah, J. B., Nixon, M. S. and Carter, J. N. (2003) Automatic Gait Recognition by Symmetry Analysis. Pattern Recognition Letters, 24: 2175-2183.

Hayfron-Acquah, J. B., Nixon, M. S. and Carter, J. N. (2002) Human Identification by SpatioTemporal Symmetry. 16th International Conference on Pattern Recognition, 11-15 August 2002, Quebec, Canada, IEEE Computer Society: 632-635.

Huang, P. S., Harris, C. J., and Nixon, M. S. (1999). "Human gait recognition in canonical space using spatio-temporal templates", IEE Proc. Vision, Image and Signal Processing, 146(2): 93-100.

Johnson, A.Y. and Bobick, A. F. (2001). A MultiView Method for Gait Recognition Using Static Body Parameters. In Josef Bigun and Fabrizio Smeraldi (eds), Springer(pub), 3rd International Conference on Audio- and Video Based Biometric Person Authentication, Halmstad, Sweden, June 6-8, 2001, 301311.

Lee, L. and Grimson, W.E.L. (2002). Gait Appearance for Recognition. In: Massimo Tistarelli, Josef Bigun and Anil K. Jain (Editors) ECCV Workshop on Biometric Authentication, Copenhagen, Denmark, June 1, 2002: 143-154.

Little, J. and Boyd, J. (1998). "Recognizing People by Their Gait: The Shape of Motion", Videre, 1(2): 1-32. 
Murase, H. and Sakai, R. (1996). "Moving object recognition in eigenspace representation: gait analysis and lip reading", Pattern Recognition Letters, 17(2): 155-162.

Niyogi, S. A. and Adelson E. H. (1994). "Analysing and recognising walking figures in xyt", In: Proceedings of the IEEE Conference on Computer Vision and Pattern Recognition, June 21-23, 1994 Seattle, USA: 469474.

Parsons, C. J. and Nixon, M. S.(1999). "Introducing focus in the generalised symmetry operator", IEEE Signal Processing Letters, 3: 49-51.

Reisfeld, D., Wolfson, H. and Yeshurun, Y. (1995). "Context-free attentional operators: The generalised symmetry transform". International Journal of Computer Vision, 14(2): 119-130.

Sadeghi, H., Allard, P., Prince, F., Labelle, H. (2001), Symmetry and limb dominance in able-bodied Gait: a review. Gait and Posture, 12 (1): 34 - 45.
Shutler, J. D., Nixon, M. S. and Harris, C. J. (2000). "Statistical gait recognition via temporal moments". Proceedings of the 4th IEEE Southwest Symposium on Image Analysis and Interpretation: IEEE Computer Society, Washington, DC, USA, April 2-4, 2000, 291-295.

Shutler, J. D., Grant, M., Nixon, M. S. and Carter, J. N. (2002) “On a Large Sequence-Based Human Gait Database. In Proceedings of the 4th International Conference on Recent Advances in Soft Computing”, November 2002, Nottingham UK: 66-72.

Tanawongsuwan, R. and Bobick, A. (2003). Performance Analysis of Time-Distance Gait Parameters under Different Speeds. In 4th International Conference on Audio- and Video Based Biometric Person Authentication, Guildford, UK, June 9-11: 715-724. 